\title{
Understanding Nasal Bone Fractures and Its Effects on Management: A Prospective Study
}

\author{
${ }^{1}$ Nayanjyoti Sarma, ${ }^{2}$ Abhinandan Bhattacharjee
}

\begin{abstract}
Objective: To evaluate mode of injury and different management options reliant on types of nasal bone fractures.
\end{abstract}

Study design: Prospective analytical study.

Subjects and methods: Seventy-five patients with nasal bone fractured were clinically and radiologically evaluated and divided into different types according to Lee's classification. They were provided conservative, closed reduction and/or osteotomy according to the type of fracture and outcome noted.

Results: Lee's classification is found to be valid in real patient scenarios and using good radiological evaluation better postoperative outcome can be achieved. For type 1 and 3 fractures, closed reduction and osteotomy are the treatment of choice.

Conclusion: We confirm that systematic approach on Lee's classification for nasal bone fracture can be used to select appropriate management strategy.

Keywords: Fracture nasal bone, Osteotomy, Lee's classification of nasal fractures, Closed reduction.

How to cite this article: Sarma N, Bhattacharjee A. Understanding Nasal Bone Fractures and Its Effects on Management: A Prospective Study. Clin Rhinol An Int J 2014;7(2):58-60.

Source of support: Nil

Conflict of interest: None declared

\section{INTRODUCTION}

Nasal bone fracture accounts for approximately $40 \%$ of faciomaxillary bone fractures. The presentation and the management issues are simple yet varied. However, there is no universally accepted classification for such fractures and only physical examination cannot determine the complexity of these fractures. This leads to a clinical dilemma in selecting the appropriate management. Classifying nasal bone fractures with respect to the force sustained and fractures detected radiologically can provide insight to appropriate

\footnotetext{
${ }^{1}$ Postgraduate Trainee, ${ }^{2}$ Assistant Professor

1,2Department of ENT, Silchar Medical College, Silchar Assam, India
}

Corresponding Author: Abhinandan Bhattacharjee, Assistant Professor, Department of ENT, Silchar Medical College, Silchar Assam, India, Phone: 03612512349, e-mail: dr.bhatta2013@ gmail.com management. Michael Lee's classification on nasal bone fracture has been adopted for the patients in our region. This has been correlated with type of injury, duration of presentation and management issues. As no such study in this aspect is available, our study intends to throw new light in the management of nasal bone fracture.

\section{AIMS AND OBJECTIVES}

- To analyze the mode of injury leading to different types of nasal bone fractures.

- To assess the outcome of different management options in relation to the types of nasal bone fractures.

\section{MATERIALS AND METHODS}

A prospective study of 75 patients of nasal trauma was conducted between July 2012 and June 2013 at Department of ENT, Silchar Medical College, Assam. Patients were specifically evaluated for side and type of fracture by single observer using conventional X-ray nasal bone AP and lateral views in addition to $\mathrm{CT}$ scan faciomaxillary region.

\section{Exclusion Criteria}

1. Nasal bone fracture in addition to other facial bones which are not part of nasal vault.

2. Nasal bone manipulation or any surgery done before presentation.

3. History of nasal trauma of more than 1 month.

On the basis of X-ray findings, correlated with CT scan, Lee's classification was followed as under:

- Type 1: Single fracture of either cartilage or bone not involving osseocartilaginous junction.

- Type 2: Fractures involving osseocartilaginous junction.

- Type 3: Multiple fractures at any location.

Septum not displaced off the spine were classified as subset A and those displaced were classified as subset B (Fig. 1).

Fracture sites taken into consideration were nasal bone, frontal process of maxilla, maxillary process of frontal bone, septum, those involving both cartilage and bone and those with comminuted fracture. Fracture cases were treated by closed reduction and/or osteotomy and the successful outcome was clinically assessed from relief of symptoms post-treatment. Data were analyzed with regards to mode of 


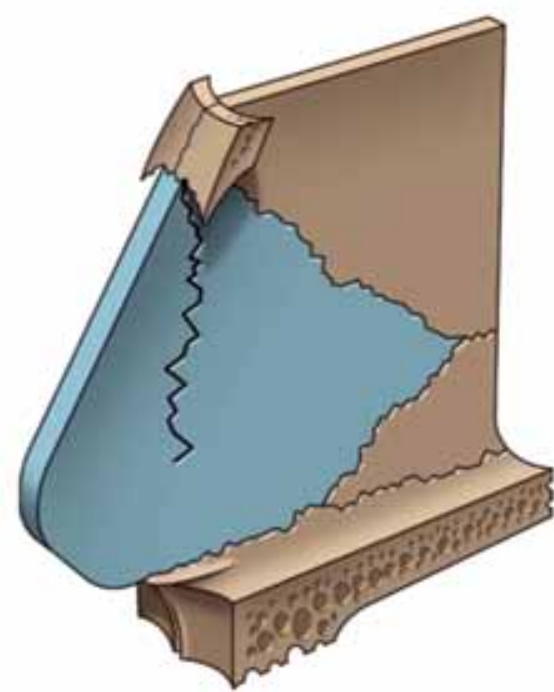

Type 1

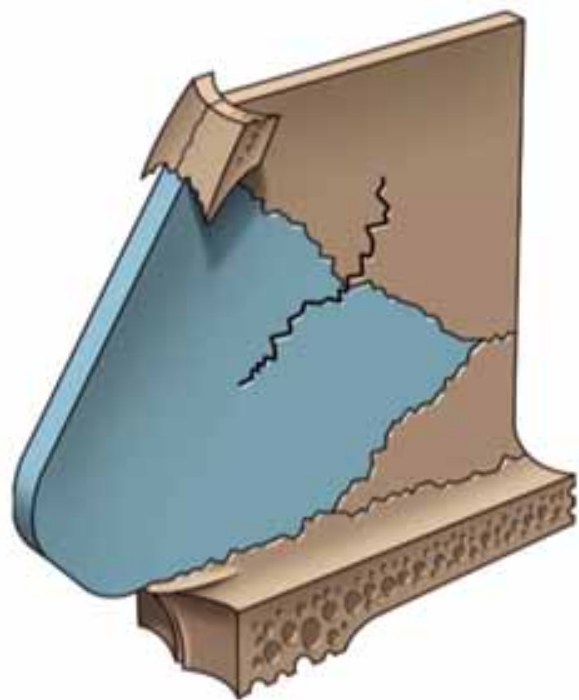

Type 2

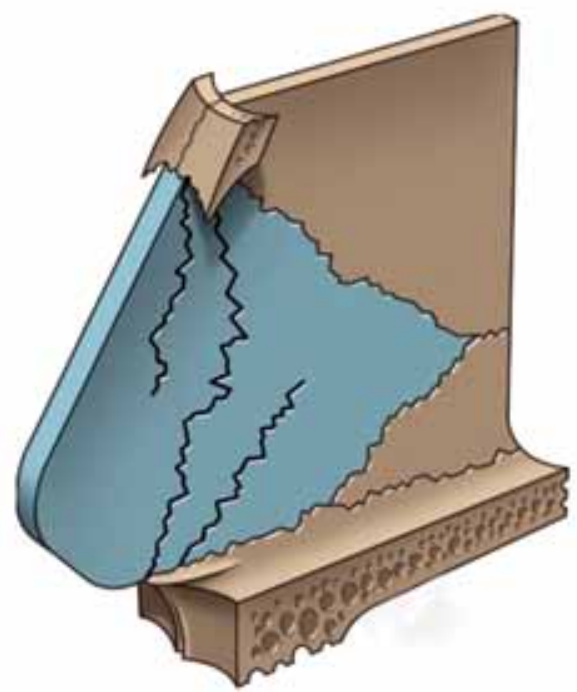

Type 3

Fig. 1: Classification of nasal fractures based on X-ray findings (Lee's classification)

injury, clinical presentation, age of injury, type of fracture and management outcome. Statistical analysis using Chisquare test was used to find out significance. p-value less than 0.005 is regarded as significant.

\section{RESULTS}

The commonest age group affected were males in 4 th decade followed by 3 rd decade with male female ratio of $4.3: 1$. The mean age in our study is 35 years.

Blow to face was the commonest cause of injury (44\%) followed by road traffic accident (34\%) presenting mainly with epistaxis (49\%) followed by pain (35\%).

Frontal blow was responsible for majority of the fractures (64\%) causing type 1a fractures followed by type $3 \mathrm{a}$ fractures. Trauma from tangential force resulted in type 1a fracture commonly (42.3\%). The most common site of fracture was nasal bone $(65 \%)$ followed by frontal process of maxilla (12\%).

The commonest indication for surgery was nasal obstruction (57\%) seen commonly in type 1 fracture while both obstruction and cosmesis $(30 \%)$ required surgical management in type 3 fractures. Epistaxis though associated with $49 \%$ was not a common indication for surgery.

Treatment outcome was best achieved for those presenting with less than 3 days history with $91.2 \%$ successful outcome.
Both closed reduction and osteotomy with or with out CR gave $90 \%$ successful outcome.

Closed reduction was done in $81.4 \%$ of type 1a cases. Osteotomy was done in $14.8 \%$ of $1 \mathrm{a}, 7 \%$ of type $2 \mathrm{a}$ and $4 \%$ of type 3 a cases. Type $B$ fractures in general did not require osteotomy. Conservative treatment was given in 1 to $5 \%$ of cases in all types. Combination of closed reduction and osteotomy was done in mostly type $3 \mathrm{a}(36.3 \%)$, followed by type $2 \mathrm{a}$ and $3 \mathrm{~b}$ (Table 1 ).

While assessing the successful treatment outcome, our results show that type la fractures (97\%) showed the highest successful outcome who received closed reduction followed by type $3 \mathrm{a}$ fracture $(88.8 \%)$ which require a combination of both closed reduction and osteotomy. Type $3 b$ fractures showed the poorest outcome (44.4\%). Among type 2 fractures, type 2 a has better outcome ( $80 \%)$.

Overall the successful outcome for closed reduction was $90 \%$, those undergoing osteotomy or a combination of osteotomy and closed reduction was $85 \%$ and those treated conservatively was $57 \%$.

\section{DISCUSSION}

Our study reported middle aged male predominance $(81 \%)$ as seen in several recent studies. ${ }^{1-3}$

Facial bones of children are more resistant to fracture due to higher elasticity of the cartilaginous component of

Table 1: Treatment modality for each type of fracture and its outcome

\begin{tabular}{lllllllll}
\hline & $1 a$ & $1 b$ & $2 a$ & $2 b$ & $3 a$ & $3 b$ & $\begin{array}{l}\text { Aims } \\
\text { achieved }\end{array}$ & $\begin{array}{l}\text { Not } \\
\text { achieved }\end{array}$ \\
\hline CR & $22(81.4 \%)$ & $3(100 \%)$ & $8(57.1 \%)$ & $0(0 \%)$ & $13(59 \%)$ & $4(50 \%)$ & $45(90 \%)$ & $5(10 \%)$ \\
O + CR & $4(14.8 \%)$ & $0(0 \%)$ & $5(35.7 \%)$ & $0(0 \%)$ & $8(35.3 \%)$ & $3(37.5 \%)$ & $18(90 \%)$ & $2(10 \%)$ \\
CON & $1(3.7 \%)$ & $0(0 \%)$ & $1(7.1 \%)$ & $1(100 \%)$ & $1(4.5 \%)$ & $1(12.5 \%)$ & $5(100 \%)$ & $0(0 \%)$ \\
\hline
\end{tabular}

Cr: Closed reduction; O + CR: Open and closed reduction; CON: Conservative management 
the nasal vault, low face to head volume ratio, and lack of sinus pneumatization., ${ }^{4,6}$

Blow to the face from violence was the commonest cause of injury to the nose due to its prominent central position and delicacy of nasal bone. While Yakub et $\mathrm{al}^{5}$ reported pain, we found epistaxis the most common presentation. Frontal impacts leads commonly type 1a and $3 a$ fractures than type 2. However, in tangential force injuries, all types of fractures are seen with type 1a being the commonest. This is because osseocartilaginous junction is in sagittal plane and so tangential force obviously leads to traumatic involvement of such joints. In accordance with Kim SH et al, we also found fracture of nasal bone commonest followed by frontal process of maxilla and the incidence decreases with age.

Nasal bone being relatively weaker and the most prominent projection in the facial region receives greater amount of force and is therefore more prone to fracture. The force from the nasal bone is transmitted to the frontal process of maxilla which makes it the second most commonest site of fracture. The relatively less incidence of concomitant fracture of septal cartilage is due to its remote location, protection by bony nasal vault and elasticity. The thick and stout maxillary process of the frontal bone is also rarely associated with fracture.

We found that as type 1 fractures deals with a single fracture it leads to more cosmetic problem esthetically but gross distortion of nasal architecture is seen in type 3 fractures where there are multiple site fractures. Therefore, nasal obstruction and cosmesis requires operative management. We also found that in old cases, results are poorer which is due to repair process causing difficulty in reposition, callus formation, hematoma, organization, fibrosis and loss of normal biological properties of injured tissues.

Conservative treatment was given when mucosal edema or minimal nasal septal deformity was seen while closed reduction with forceps and osteotomy was based on type of fracture. Fattahi et al showed closed reduction to be an effective method of treatment as long as careful attention is paid to the key regions in the nasal complex, including the septum at the initial time of treatment. ${ }^{7}$ However, Staffel JG concluded that individually tailored protocol of closed reduction with other operative strategies gave better results than by closed reduction alone. ${ }^{8}$

Our study found that type 1 fractures with communition gives marginally poorer result. When fractures involve osseocartilaginous junction (type 2), both closed and open reduction was required in half of cases with high success rates in type $2 \mathrm{a}$ than $2 \mathrm{~b}$ fractures. Similarly, type $3 \mathrm{a}$ fractures showed high successful outcome than type $3 \mathrm{~b}$. However, more extensive procedures like osteotomy were used for type 3b. Murray JA et al indicated that open reduction is the preferred treatment for patients with a deviation of more than half the bridge width of the nose. ${ }^{9}$ Therefore, fractures without communition in general gives better results. The treatment modality for type 1 and 3 was seen to be quite clear cut but for type 2 fractures there is dilemma between closed and open reduction.

Although Lee proposed his classification based on cadaver dissection, we applied this in real patient scenario and used radiological means to confirm details of fractures. We confirm that this classification can be used to select appropriate management strategy.

\section{CONCLUSION}

Although nasal bone fractures are common, management issues are less clear. Lack of proper classification of such fractures leads to problems in selecting appropriate treatment options. Lee's classification can be applied in real patient scenarios and we concluded that frontal force generally leads to type 1 and 3 for which closed reduction and osteotomy are the treatment of choice respectively. Interestingly, type 2 fractures are from tangential force and further in depth study is required to decide more on the appropriate treatment modality for this type of fractures. Also, type B has poorer results than type A.

Therefore, systematic approach using Lee's fracture classification using good radiological evaluation provides appropriate management and better clinical outcome.

\section{REFERENCES}

1. Gassner R, Tuli, Hachl O, et al. Craniomaxillofacial trauma in children: a review of 3,385 cases with 6,060 injuries in 10 years. J Oral Maxillofac Surg 2004;62:399-340.

2. Lida S, Matsuya T. Paediatric maxillofacial fractures: their aetiological characters and fracture patterns. J Craniomaxillofac Surg 2002;30:237-241

3. Sherick DG, Buchman SR, Patel PP. Paediatric facial fractures: a demographic analysis outside an urban environment. Ann Plast Surg 1997;38:578-584.

4. Murphy RX Jr, Birmingham KL, Okunski WJ, et al. Influence of restraining devices on patterns of paediatric facial trauma in motor vehicle collisions. Plast Reconst Surg 2001;107:34-37.

5. Yakup CIL, Kahraman E. An analysis of 45 patients with pure nasal fractures. Turkish J Trauma Emerg Surg 2013;19(2): 152-156.

6. Kim SH, Lee SH, Cho PD. Analysis of 809 facial bone fractures in paediatric and adolescent population. Arch Plast Surg 2012 Nov;39(6):606-611.

7. Fattahi T, et al. Repair of nasal complex fractures and the need for secondary septorhinoplasty. J Oral Maxillofac Surg 2006 Dec;64(12):1785-1789.

8. Staffel JG, et al. Optimizing treatment of nasal fractures. Laryngoscope 2002 Oct;112(10):1709-1719.

9. Murray JA, et al. Open vs closed reduction of the fractured nose. Arch Otolaryngol 1984 Dec;110(12):797-802. 